multiple cycles. This process is completely automated through the CODEX $^{\circledast}$ instrument and readily deployable on commercially available fluorescence microscopy systems. Using a $30+$ antibody CODEX ${ }^{\varpi}$ panel, we compared formalin-fixed paraffin embedded (FFPE) human breast cancer tissues at different stages of disease progression with normal breast tissues. Our antibody panel was designed to detect cancer cells as well as non-malignant cells in order to comprehensively survey the tumor microenvironment and normal control tissues. Data were analyzed using the $\operatorname{CODEX}^{\circledast}$ software suite to identify key cell types and analyze spatial associations.

Results Our analyses revealed more than 20 distinct cell types in human breast cancer and normal tissues. Cell populations, biomarker expression and cellular spatial distributions differed distinctly between cancerous and normal breast tissues. Differences were robust, repeatedly observed and indicative of altered cellular milieus in normal versus cancerous breast tissues.

Conclusions Collectively, these data establish $\operatorname{CODEX}^{\circledast}$ as a readily deployable and practical tool for spatially-resolved, highly multiplexed biomarker analysis of human FFPE samples.

Disclosure Information O. Braubach: A. Employment (full or part-time); Significant; Akoya Biosciences. S. Basak: A. Employment (full or part-time); Significant; Akoya Biosciences. M. Gallina: A. Employment (full or part-time); Significant; Akoya Biosciences. W. Lee: A. Employment (full or part-time); Significant; Akoya Biosciences. J. Kim: A. Employment (full or part-time); Significant; Akoya Biosciences. C. Hempel: A. Employment (full or part-time); Significant; Akoya Biosciences. E. Williams: A. Employment (full or part-time); Significant; Akoya Biosciences. O. Shang: A. Employment (full or parttime); Significant; Akoya Biosciences. B. Cheung: A. Employment (full or part-time); Significant; Akoya Biosciences. J. Kennedy-Darling: A. Employment (full or part-time); Significant; Akoya Biosciences.

\section{P01.07 TARGETING A MEMBRANE PROXIMAL EPITOPE ON MESOTHELIN INCREASES THE TUMORICIDAL ACTIVITY OF A BISPECIFIC ANTIBODY BLOCKING CD47 ON TUMOR CELLS}

E Hatterer*, X Chauchet, F Richard, L Barba, V Moine, L Chatel, N Fischer, W Ferlin, V Buatois, K Masternak, L Shang. Light Chain Biosciences/Novimmune, Plan les ouates Geneva, Switzerland

\subsection{6/jitc-2020-ITOC7.20}

Background Mesothelin (MSLN) is recognized as a relevant tumor-associated antigen for cancer immunotherapy, because of its overexpression on various solid tumors, including mesothelioma, pancreatic, lung, gastric and ovarian carcinoma. However, an anti-MSLN monoclonal antibody (mAb), amatuximab, has demonstrated only limited efficacy in clinical trials. It has been already demonstrated that the targeting of a membrane-distal domain of an antigen with a mAb is suboptimal at inducing Fc-related effector functions. As amatuximab targets a membrane-distal domain of MSLN, we investigated whether mAbs targeting different epitopes would bestow a better efficacy. Furthermore, in order to incorporate novel modalities to enhance tumor-killing, we have paired these MSLN targeting arms with an anti-CD47 arm to generate bispecific antibodies (bsAb). Indeed, the 'don't eat me signal' CD47 is a promising target in cancer and therapeutic blockade has recently showed clinical evidence of efficacy. Therefore, we investigated the contribution of a CD47 arm and the impact of the different anti-MSLN targeting arms on the tumoricidal activities of CD47xMSLN bsAbs.

Materials and Methods A panel of anti-MSLN mAbs and CD47xMSLN biAbs carrying the same anti-CD47 arm and different anti-MSLN arms were generated and characterized for their epitope specificity. Their tumor cell killing efficacy in vitro and in vivo was analyzed using cell-based assays, xenograft models and various MSLN+ human malignant cell lines originated from different tissues (e.g., lung, gastric and hepatic origin).

Results Our data revealed that all CD47xMSLN bsAbs, regardless of the recognized MSLN epitope, showed higher activity than the corresponding anti-MSLN mAbs in tumor-cell killing assays and demonstrated superior anti-tumor activity in a xenograft model. Targeting a membrane-proximal epitope rendered an anti-MSLN mAb more effective in mediating antibody-dependent cell-mediated cytotoxicity (ADCC) but did not optimize antibody dependent cellular phagocytosis (ADCP) activity. However, targeting the membrane-proximal epitope of MSLN afforded the CD47xMSLN bsAb enhanced ADCC and ADCP activity, resulting in superior activity in vivo. Mechanistically, engaging a MSLN membrane proximal region with a CD47-bsAb format not only enhanced FcrR-IIIA signaling but also interestingly disrupted more efficiently the CD47/SIRP $\alpha$ axis, resulting in optimized phagocytosis of tumor cells. Finally, we showed that treatment with CD47xMSLN bsAb targeting membrane proximal MSLN epitope induced an accumulation of myeloid cells and NK cells in the tumor microenvironment.

Conclusions This study demonstrated that when designing antibody-based molecules, the targeted region on a tumor-associated antigen needs to be carefully considered to ensure maximal effector function. In the context of MSLN-positive solid tumors, we showed that an approach targeting a membrane-proximal epitope coupled to a CD47-blocking arm afforded an improved ADCC and ADCP profile, translating into increased in vivo efficacy.

Disclosure Information E. Hatterer: None. X. Chauchet: None. F. Richard: None. L. Barba: None. V. Moine: None. L. Chatel: None. N. Fischer: None. W. Ferlin: None. V. Buatois: None. K. Masternak: None. L. Shang: None.

\section{P01.08 BEYOND PD-1: CHARACTERIZATION OF NEW CHECKPOINTS RESTRICTING FUNCTION OF CYTOTOXIC LYMPHOCYTES INFILTRATING HUMAN CARCINOMA}

${ }^{1}$ AS Herbstritt", ${ }^{1} \mathrm{PU}$ Prinz, ${ }^{2} \mathrm{M}$ Maxwell, ${ }^{2} \mathrm{M}$ Kadiyala, ${ }^{2} \mathrm{D}$ Yan, ${ }^{1} \mathrm{E}$ Noessner. ${ }^{1}$ HelmholtzZentrum München, Immunoanalytics, München, Germany; ${ }^{2}$ Phio Pharmaceuticals, Marlborough, MA, USA

\subsection{6/jitc-2020-ITOC7.21}

Background T and NK cells from human renal cell carcinoma (RCC) are functionally non-responsive. Analysis of the TCR signaling cascade required for effector function identified that proximal signaling molecules were activated whereas activation of downstream ERK was blocked. Further investigation showed increased diacylglycerol kinase alpha (DGK- $\alpha$ ) levels in $\mathrm{T}$ and NK cells from the RCC tumor microenvironment (TME). These cells were refractory to stimulation showing no degranulation or IFN- $\gamma$ production. Using a small molecule DGK- $\alpha$ inhibitor (R59022), the function of tumor-infiltrating 